\title{
谷 林
}

理化学研究所革新知能統合研究センター

研究員

\section{解釈可能なインタラクティブ深層学習}

\section{$\S 1$. 研究成果の概要}

In 2019, I was working on the foundation of this project including the gaze estimation and medical image diagnose.

The gaze estimation attempts to guess the point of gaze where people would look. The gaze provides a unique insight into human's information processing behaviour. The research on gaze estimation aims to build the relation between the tracked gaze and the medical images. To gain this understanding, the first step is to estimate the gaze position where doctors would see when inspecting the medical image. Specifically, we design a novel deep learning model to estimate the spatial attention distribution on image by automatically identifying the symptom and other suspicious area in the image.

In the meantime, it is also necessary to explore the diagnose mechanism of artificial intelligence. In 2019, we focus on the liver cancer and retinal image. For the liver cancer, we propose a novel 3D CNN based diagnose algorithm to predict the severity grade of tumour from the contrast enhanced MRI images. This algorithm has delivered a high accuracy and could save the labour of doctors from checking the MRI images. In the meantime, we are also analysing the morphology features of the vessel. When there is a disease, the surrounding vessels would also be affected due to the physiological condition. Here, we utilise several cutting edge tools to measure the statistical relation and explore the pathological mechanism behind this relation. 